\title{
PPAR $\gamma$ agonism increases rat adipose tissue lipolysis, expression of glyceride lipases, and the response of lipolysis to hormonal control
}

\author{
W. T. Festuccia • M. Laplante $\cdot$ M. Berthiaume • \\ Y. Gélinas • Y. Deshaies
}

Received: 10 March 2006 / Accepted: 2 May 2006 / Published online: 14 August 2006

(C) Springer-Verlag 2006

\begin{abstract}
Aims/hypothesis The aim of this study was to investigate the effect and mechanisms of action of in vivo peroxisome proliferator-activated receptor $\gamma$ (PPAR $\gamma$ ) activation on white adipose tissue (WAT) lipolysis and NEFA metabolism.

Materials and methods Study rats were treated for 7 days with $15 \mathrm{mg} / \mathrm{kg}$ of rosiglitazone per day; control rats were not treated. After a 6-h fast, lipolysis and levels of mRNA for lipases were assessed in explants from various adipose depots.

Results Rosiglitazone markedly increased basal and noradrenaline (norepinephrine)-stimulated glycerol and NEFA release from WAT explants, and amplified their inhibition by insulin. Primary adipocytes isolated from PPAR $\gamma$
\end{abstract}

Electronic supplementary material Supplementary material is available for this article at http://dx.doi.org/10.1007/s00125-0060336-y and is accessible for authorized users.

The first two authors contributed equally to the work reported in this manuscript.

W. T. Festuccia $\cdot$ M. Laplante $\cdot$ M. Berthiaume $\cdot$ Y. Gélinas •

Y. Deshaies

Laval Hospital Research Center, Faculty of Medicine,

Laval University,

Quebec G1V 4G5, Canada

W. T. Festuccia $\cdot$ M. Laplante $\cdot$ M. Berthiaume $\cdot$ Y. Gélinas $\cdot$

Y. Deshaies

Department of Anatomy and Physiology, Faculty of Medicine,

Laval University,

Quebec G1V 4G5, Canada

Y. Deshaies $(\triangle)$

Laval Hospital Research Centre, Laval Hospital,

d'Youville Y3110, 2725 Ch Sainte-Foy,

Quebec G1V 4G5, Canada

e-mail: yves.deshaies@phs.ulaval.ca agonist-treated rats were also more responsive to noradrenaline stimulation expressed per cell, ruling out a contribution of an altered number of mature adipocytes in explants. Rosiglitazone concomitantly increased levels of mRNA transcripts for adipose triglyceride lipase (ATGL) and monoglyceride lipase (MGL) in subcutaneous and visceral WAT, and mRNA for hormone-sensitive lipase (HSL) in subcutaneous WAT. Lipase expression increased within $12 \mathrm{~h}$ of in vitro exposure of naïve explants to rosiglitazone, suggesting direct transcriptional activation. In parallel, chronic in vivo treatment with rosiglitazone lowered plasma NEFAs and exerted in WAT its expected stimulatory action on glycerol and NEFA recycling, and on the expression of genes involved in NEFA uptake and retention by WAT, such processes counteracting net NEFA export.

Conclusions/interpretation These findings demonstrate that, in the face of its plasma NEFA-lowering action, PPAR $\gamma$ agonism stimulates WAT lipolysis, an effect that is compensated by lipid-retaining pathways. The results further suggest that PPAR $\gamma$ agonism stimulates lipolysis by increasing the lipolytic potential, including the expression levels of the genes encoding adipose triglyceride lipase and monoglyceride lipase.

Keywords Adipose triglyceride lipase - Desnutrin . Hormone-sensitive lipase · Insulin · Lipolysis . Monoglyceride lipase $\cdot$ NEFA $\cdot$ Noradrenaline . PPAR $\gamma$ agonism $\cdot$ Rosiglitazone
Abbreviations
ATGL adipose triglyceride lipase
DBcAMP dibutyryl cyclic AMP
EPI epididymal adipose depot
ESM Electronic supplementary material
FABP4 adipose fatty acid binding protein 4 
FATP1 fatty acid transporter protein 1

GYK glycerol kinase

HSL hormone-sensitive lipase

ING inguinal adipose depot

MES mesenteric adipose depot

MGL monoglyceride lipase

PEPCK phosphoenolpyruvate carboxykinase

$\operatorname{PPAR} \gamma \quad$ peroxisome proliferator-activated receptor $\gamma$

RETRO retroperitoneal adipose depot

WAT white adipose tissue

\section{Introduction}

Thiazolidinediones are used in the treatment of insulinresistant states. The compounds exert their potent insulinsensitising action by interacting with, and activating, peroxisome proliferator-activated receptor $\gamma$ (PPAR $\gamma)$, a nuclear receptor that is mainly expressed in adipose tissue. Along with their effects on white adipose tissue (WAT) adipokine production and extra-adipose actions [1, 2], several lines of evidence suggest that one of the insulinsensitising mechanisms of thiazolidinediones is a reduction in circulating NEFAs, which protects non-adipose tissues against lipid overload and consequent insulin resistance (reviewed in [2]).

Plasma concentrations of NEFAs represent the balance between their release from WAT and uptake by oxidative tissues, with a contribution from adipose reuptake. All of these processes are affected by thiazolidinediones. Indeed, rosiglitazone has been shown to reduce NEFA uptake by the liver and muscle [3], and to increase NEFA uptake, intracellular re-esterification, and storage into triglycerides in WAT [4]. Because of its effect on plasma NEFAs, PPAR $\gamma$ agonism might also be expected to reduce lipolysis; however, previous studies investigating this issue have produced conflicting results. Whereas some studies have reported lower in vitro rates of glycerol and NEFA release from WAT $[5,6]$, others that include in vivo NEFA kinetic approaches [4, 7] suggest that PPAR $\gamma$ agonism stimulates lipolysis, an effect that would be counteracted by its concomitant action on NEFA reuptake and re-esterification.

Until recently, the hydrolysis of triglycerides in WAT was thought to be catalysed exclusively by the enzyme hormone-sensitive lipase (HSL). Lipolytic hormone-stimulated, protein kinase A-mediated phosphorylation of both HSL and perilipin results in hydrolysis of triglyceride into NEFAs and monoglyceride, which is further converted to NEFA and glycerol by monoglyceride lipase (MGL) [8]. Recent studies showing that adipocytes from HSL knockout mice retain a marked basal and adrenergically stimulated lipolysis and accumulate diglycerides after stimulation of lipolysis demonstrated that HSL is not the sole lipase involved in triglyceride catabolism [9-11]. Three independent groups have discovered a protein-alternatively termed adipose triglyceride lipase (ATGL) [12], desnutrin [13] or calcium-independent phospholipase $\mathrm{A}_{2}$ /lipase $\zeta$ $\left(\mathrm{iPLA}_{2} \zeta\right)$ [14] - that fulfils all the predicted characteristics of this new lipase: a preference for the hydrolysis of the first triglyceride ester bond, drastically reduced lipolysis following antibody or antisense ATGL inhibition, and enhanced basal and isoproterenol-stimulated lipolysis following $A T G L$ overexpression [15]. ATGL therefore plays a particularly important role in the control of basal lipolysis, but also appears to participate in the lipolytic response to adrenergic stimulation [16].

The characterisation of ATGL prompted us to consider the possibility that, if PPAR $\gamma$ agonism indeed enhances lipolysis, it may do so partly via altering lipase expression. The present study therefore tested the hypothesis that thiazolidinediones increase WAT lipolysis, through assessment of the impact of chronic rosiglitazone treatment of rats on NEFA and glycerol release by WAT explants. Because thiazolidinediones exert depot-specific actions [17] and their putative modulation of $A T G L$ and $M G L$ expression has not yet been addressed, four white adipose depots representing visceral and subcutaneous fat were investigated. Noradrenaline (norepinephrine)-induced stimulation and insulin-induced inhibition of lipolysis were assessed to establish the impact of PPAR $\gamma$ agonism on the response of lipolysis to neuroendocrine/metabolic control. The depotspecific levels of $A T G L, H S L$ and $M G L$ mRNAs were determined to verify the second hypothesis: that rosiglitazone-induced changes in lipolysis are associated with the expression levels of its obligate lipases.

\section{Materials and methods}

Animals and treatment Male Sprague-Dawley rats (200225 g, Charles River Laboratories, St Constant, QC, Canada) were housed individually in stainless steel cages $\left(23 \pm 1^{\circ} \mathrm{C}, 12 \mathrm{~h} / 12 \mathrm{~h}\right.$ light/dark cycle, lights on at $\left.12.00 \mathrm{~h}\right)$. The Principles of Laboratory Animal Care (NIH publication no. 85-23, revised 1985) were followed, and animal care and handling were performed in accordance with the Canadian Guide for the Care and Use of Laboratory Animals. All experimental procedures received prior approval by Laval University's animal protection committee. Rats were divided into a control group and a treated group that was given the PPAR $\gamma$ agonist rosiglitazone (purchased as Avandia GlaxoSmithKline, Mississauga, ON, Canada at a local pharmacy) at a daily dose of $15 \mathrm{mg} / \mathrm{kg}$ body weight as an admixture to the food (Charles River Rodent Diet 
\#5075; Ralston Products, Woodstock, ON, Canada) for 1 week. This dose is slightly above that which elicits the maximal response of glucose and lipid metabolism to rosiglitazone in rats, as determined in pilot studies. For all experimental procedures described below, food was removed at $07.00 \mathrm{~h}$, and rats were killed by decapitation after $6 \mathrm{~h}$ of fasting.

In vitro basal, NA-stimulated, and insulin-inhibited lipolysis Adipose explants (20-25 mg) of inguinal (ING), retroperitoneal (RETRO), epididymal (EPI) and mesenteric (MES) WAT were incubated in $1 \mathrm{ml}$ of Krebs Ringer bicarbonate buffer of the following composition (in $\mathrm{mmol} / \mathrm{l}$ ): $118 \mathrm{NaCl}, 4.8 \mathrm{KCl}, 1.25 \mathrm{CaCl}_{2}, 1.2 \mathrm{KH}_{2} \mathrm{PO}_{4}, 1.2 \mathrm{MgSO}_{4}$, $25 \mathrm{NaHCO}_{3}, 5$ glucose, supplemented with $2.5 \%$ NEFAfree BSA (Sigma, Oakville, ON, Canada), pH 7.4. Fat explants were incubated for $2 \mathrm{~h}$ in a humidified atmosphere of $5 \% \mathrm{CO}_{2}$ and $95 \% \mathrm{O}_{2}$ at $37^{\circ} \mathrm{C}$. Insulin $(100$ and $500 \mathrm{pmol} / \mathrm{l})$, noradrenaline $(1 \mu \mathrm{mol} / \mathrm{l})$ and dibutyryl cAMP (DBcAMP, $1 \mathrm{mmol} / \mathrm{l}$ ) were added to the incubation buffer to inhibit or stimulate lipolysis. At the end of the incubation, WAT explants were removed and media were frozen until the measurement, using reagent kits, of NEFA (Wako Pure Chemical Industries, Richmond, VA, USA) and glycerol (Sigma). Data for NEFA and glycerol release are expressed as nmol $\mu \mathrm{g} \mathrm{DNA}^{-1} \mathrm{~h}^{-1}$ (DNeasy Tissue Kit; Qiagen, Mississauga, ON, Canada) to correct for cell number. DNA content was determined according to the manufacturer's instructions. Additional experimental details are provided in the Electronic supplementary material (ESM text 1).

In vitro exposure of explants to rosiglitazone Minced pieces ( $40 \mathrm{mg} /$ well) of each of the four depots described above, obtained from four control, untreated, fasted rats, were incubated in $1 \mathrm{ml}$ of DMEM (Invitrogen, Burlington, ON, Canada) supplemented with pure rosiglitazone (Cayman Chemical Company, Ann Arbor, MI, USA) to a final concentration of $10 \mu \mathrm{mol} / \mathrm{l}$, or carrier DMSO for $12 \mathrm{~h}$. Treatments were performed in duplicate for each rat. Fat explants were then removed and frozen in liquid nitrogen until RNA isolation and analysis.

Triglyceride/NEFA cycling, PEPCK and GYK activities These variables were quantified according to established methods, as detailed in ESM text 2.

RNA isolation and analysis RNA was isolated from adipose depots using Qiazol and the RNAeasy Lipid Tissue Kit (Qiagen). For cDNA synthesis, Expand reverse transcriptase (Invitrogen) was used following the manufacturer's instructions and cDNA was diluted in DNase-free water (1:25) before quantification by real-time PCR. The mRNA transcript levels were measured in duplicate samples through chemical detection of the PCR products with SYBR Green I (Molecular Probes, Willamette Valley, OR, USA) using a Rotor Gene 3000 system (Montreal Biotech, Montreal, QC, Canada). Primers are listed in ESM Table 1, and additional methodological details are presented in ESM text 3 .

Serum determinations Serum glucose concentrations were measured by the glucose oxidase method with the YSI 2300 STAT Plus glucose analyser (YSI, Yellow Springs, OH, USA). Insulin levels were determined by RIA (Linco Research, St Charles, MO, USA) with rat insulin as standard. Serum triglyceride levels were measured by an enzymatic method (Roche Diagnostics, Montreal, QC, Canada), and serum NEFAs and glycerol were determined enzymatically as described above.

Statistical analysis Data are expressed as means \pm SEM. Simple effects of rosiglitazone treatment were analysed by Student's unpaired $t$-test. Where appropriate, factorial ANOVA followed by Newman-Keuls multiple range test were used to compare the effects of rosiglitazone in various adipose depots. A $p$ value $<0.05$ was used as the threshold of significance.

\section{Results}

Final body weight and body weight gain were significantly increased (6\% and $50 \%$, respectively) after 7 days of rosiglitazone treatment (Table 1), higher body weight being associated with an increase in both food intake $(18 \%)$ and food efficiency $(23 \%)$, and in the weight of all WAT depots $(30-35 \%)$ and interscapular brown adipose tissue (116\%). Rosiglitazone significantly reduced plasma levels of insulin $(-35 \%)$, NEFAs $(-57 \%)$, and triglycerides $(-55 \%)$, but did not alter serum glucose or glycerol levels. Rosiglitazone therefore exerted its expected metabolic actions on energy balance, WAT mass, indices of insulin sensitivity, and lipidaemia.

The effects of rosiglitazone on rates of basal and noradrenaline/DBcAMP-stimulated glycerol and NEFA release normalised per DNA are illustrated in Fig. 1. All three visceral depots behaved similarly on a qualitative basis, and only data for the RETRO and ING (subcutaneous) depots are presented. In control rats, with the exception of basal NEFA release, rates of glycerol and NEFA release were, as expected, lower in the ING (Fig. 1a,b) than in the RETRO (Fig. 1c,d) depot. Noradrenaline and DBcAMP increased glycerol and NEFA release to the same extent in both adipose depots. Also, in both depots, chronic 
Table 1 Measurements in rosiglitazone-treated and control rats

\begin{tabular}{lll}
\hline Parameter & Control rats & $\begin{array}{l}\text { Rosiglitazone-treated } \\
\text { rats }\end{array}$ \\
\hline Body weight (g) & $330 \pm 4$ & $348 \pm 3^{\mathrm{a}}$ \\
Body weight gain (g) & $47 \pm 3$ & $67 \pm 3^{\mathrm{a}}$ \\
Food intake (g) & $164 \pm 3$ & $194 \pm 4^{\mathrm{a}}$ \\
Food efficiency (\%) & $28 \pm 1$ & $35 \pm 1^{\mathrm{a}}$ \\
ING (g) & $3.2 \pm 0.2$ & $4.3 \pm 0.2^{\mathrm{a}}$ \\
RETRO (g) & $2.0 \pm 0.2$ & $2.7 \pm 0.1^{\mathrm{a}}$ \\
EPI (g) & $2.7 \pm 0.2$ & $3.5 \pm 0.2^{\mathrm{a}}$ \\
MES (g) & $2.0 \pm 0.1$ & $2.7 \pm 0.2^{\mathrm{a}}$ \\
Brown adipose tissue (g) & $0.31 \pm 0.01$ & $0.67 \pm 0.02^{\mathrm{a}}$ \\
Insulin (pmol/l) & $186 \pm 22$ & $122 \pm 18^{\mathrm{a}}$ \\
Glucose (mmol/1) & $7.9 \pm 0.3$ & $7.8 \pm 0.1$ \\
NEFAs (mmol/l) & $0.47 \pm 0.05$ & $0.20 \pm 0.07^{\mathrm{a}}$ \\
Glycerol (mmol/l) & $0.128 \pm 0.011$ & $0.121 \pm 0.003$ \\
Triglycerides (mmol/l) & $2.0 \pm 0.2$ & $1.1 \pm 0.1^{\mathrm{a}}$ \\
\hline
\end{tabular}

Data are means \pm SEM of 6-12 rats. Treated rats received rosiglitazone for 7 days

${ }^{\mathrm{a}} p<0.05$ vs controls (Student's unpaired $t$-test)

${ }^{\mathrm{b}}$ Calculated as body weight gain $(\mathrm{g})$ per $100 \mathrm{~g}$ food ingested rosiglitazone treatment markedly increased basal lipolysis, as demonstrated by the much larger release of glycerol (eight- to ten-fold) and NEFAs (five-fold) from explants of treated compared with untreated rats. Rosiglitazone retained its ability to stimulate the release of lipolytic products in the presence of noradrenaline and DBcAMP such that the lipolytic response to combined adrenergic or DBcAMP stimulation and chronic rosiglitazone treatment were additive. The possible contribution of rosiglitazone-induced changes in the number of lipolytically competent cells was excluded using isolated adipocytes, as discussed in ESM text 4 and illustrated in ESM Fig. 1.

Because PPAR $\gamma$ agonism affects adipose lipid metabolism largely via modulation of gene expression, we next sought to determine whether the rosiglitazone-induced stimulation of lipolysis was associated with changes in mRNA levels of the major intracellular glyceride lipases. In control rats, levels of $A T G L$ (Fig. 2a), HSL (Fig. 2b) and $M G L$ mRNAs (Fig. 2c) were lower in ING than in all visceral depots, with the exception of $M G L$ mRNA in MES. Concomitant with basal lipolysis, rosiglitazone increased levels of both $A T G L$ (Fig. 2a; ING, six-fold; all visceral
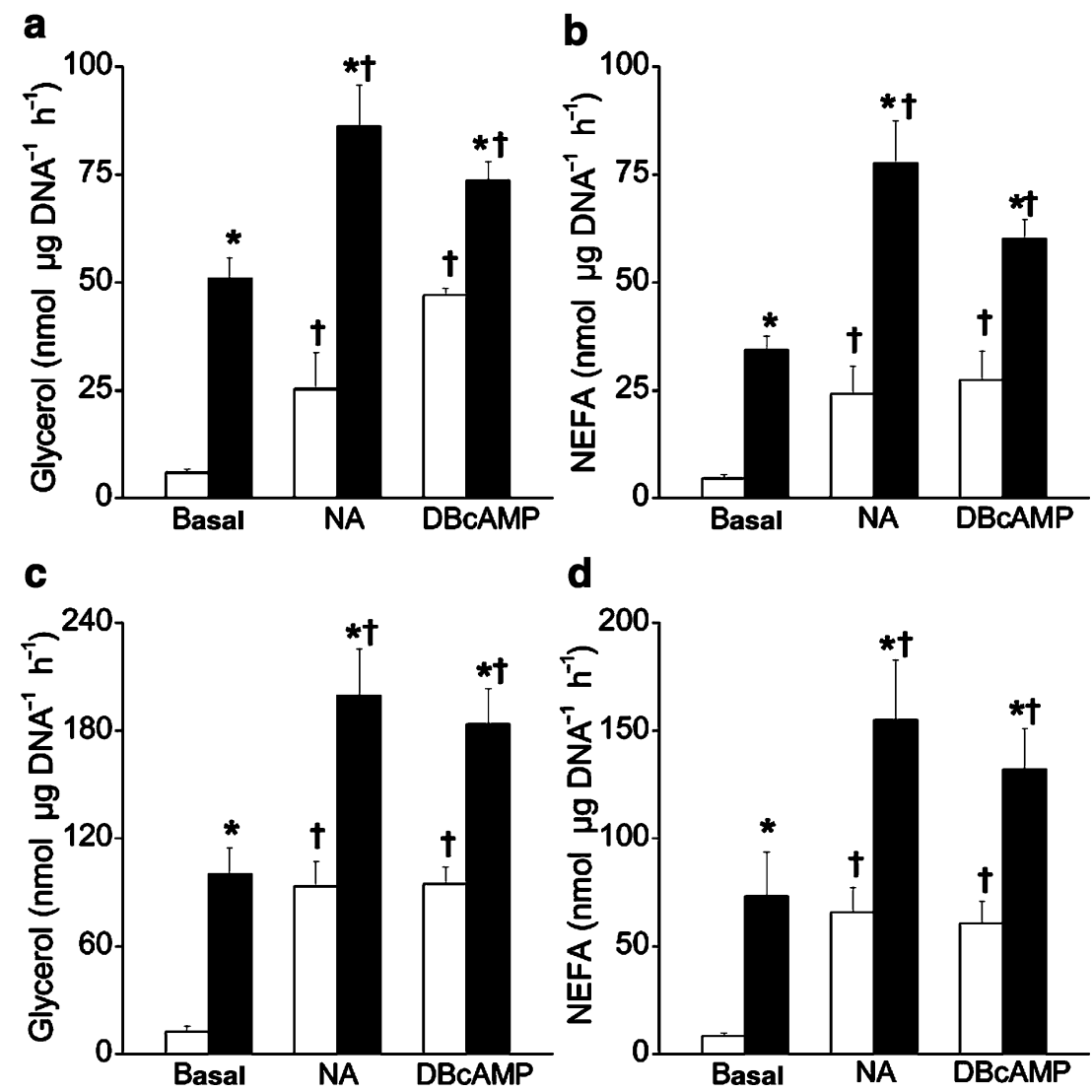

d

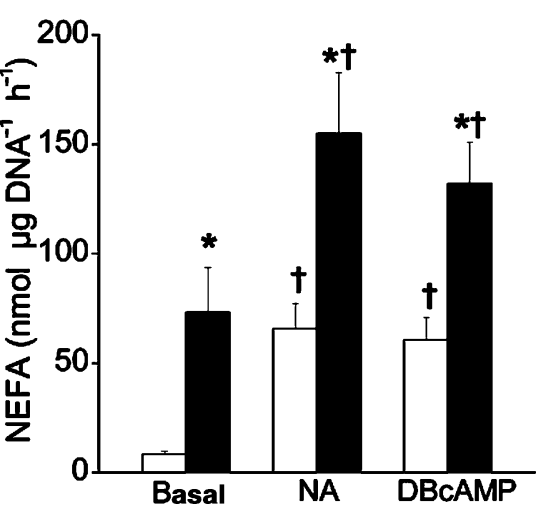

Fig. 1 In vitro basal, noradrenaline $(N A)$ - and DBcAMP-stimulated rates of glycerol $(\mathbf{a}, \mathbf{c})$ and NEFA $(\mathbf{b}, \mathbf{d})$ release by explants of ING $(\mathbf{a}, \mathbf{b})$ and RETRO $(\mathbf{c}, \mathbf{d})$ adipose depots of control (empty columns) and rosiglitazone-treated rats (filled columns). Each column represents

the mean \pm SEM for six rats. ${ }^{*} p<0.05$ vs untreated control under the same incubation conditions; $\uparrow p<0.05$ vs basal unstimulated conditions for same treatment group 

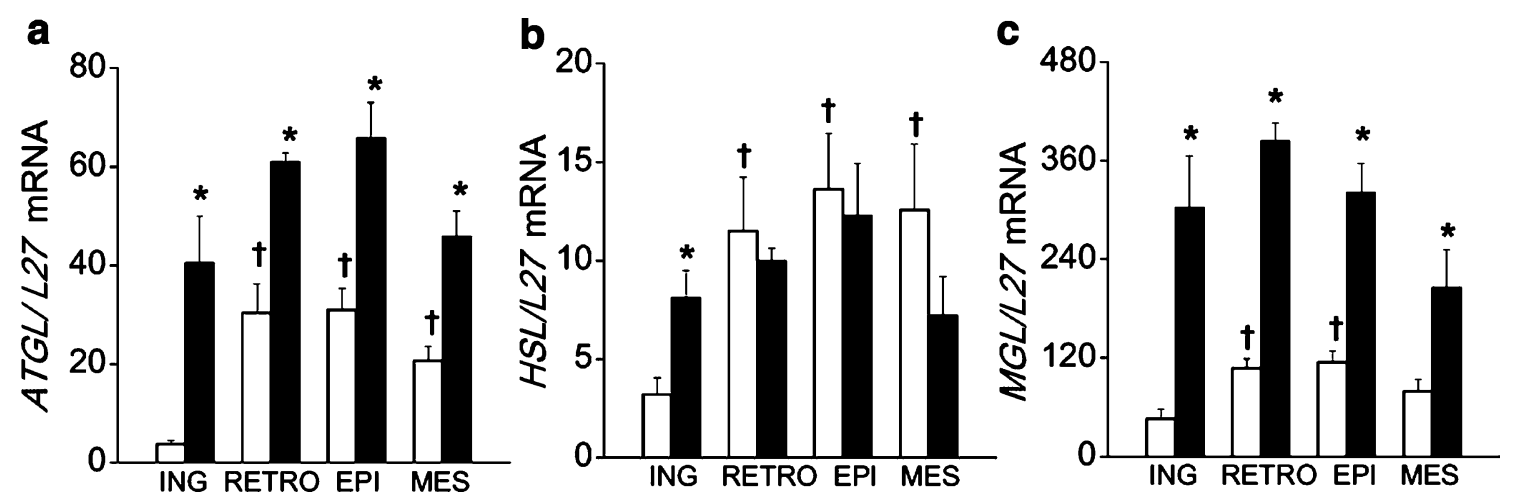

Fig. 2 Levels of $A T G L$ (a), HSL (b) and $M G L$ (c) mRNAs in ING, RETRO, EPI and MES adipose depots of control (empty columns) and rosiglitazone-treated rats (filled columns). Each column represents the mean \pm SEM for six rats. ${ }^{*} p<0.05$ vs control in the same depot; $\dagger p<0.05$ vs ING depot of control rats depots, two-fold) and $M G L$ (Fig. 2c; ING, seven-fold; all visceral depots, three- to four-fold) mRNAs. Rosiglitazone increased HSL mRNA levels (2.5-fold) only in the ING depot (Fig. 2b). With data of the four adipose depots pooled, rates of glycerol release correlated with mRNA levels of both $A T G L$ (Fig. 3a) and $M G L$ (Fig. 3c), but not with those of $H S L$ (Fig. 3b). Similar correlation coefficients were found between lipase mRNAs and NEFA release ( $r=0.59$ and $0.77, p<0.0001$, for $A T G L$ and $M G L$, respectively, and 0.07 , NS, for $H S L$ ).

To address the question of whether rosiglitazone directly stimulates lipase expression, adipose tissue explants from untreated rats were exposed for $12 \mathrm{~h}$ to the agonist, and lipase mRNA levels were compared with levels of FABP4 mRNA, which codes for adipose fatty acid binding protein 4 , a direct PPAR response element-containing target of PPAR $\gamma$ agonism. As depicted in Fig. 4, within 12 h, rosiglitazone increased the mRNA levels of all lipases to approximately the same extent as that of FABP4 in both ING and RETRO depots, without altering the mRNA level of the reference L27 gene.

As rosiglitazone proved to increase key elements of the lipolytic machinery, we next sought to determine whether the agonist altered the response of WAT to the antilipolytic action of physiological amounts of insulin. In control ING explants, insulin failed to inhibit basal lipolysis, represented by absolute glycerol release (Fig. 5a) or per cent of relative to basal (Fig. 5b), but did reduce NEFA release (Fig. 5c,d) by virtue of its stimulatory action on NEFA recycling. In ING explants of rosiglitazone-treated rats, insulin inhibited the release of both glycerol and NEFAs, and to a much greater extent than in control explants (Fig. 5a-d). In the RETRO depot, insulin reduced both glycerol (Fig. 5e,f) and NEFA release (Fig. $5 \mathrm{~g}, \mathrm{~h}$ ), regardless of treatment, and the magnitude of the inhibition was again greater in explants from rosiglitazone-treated than control rats. Notably, despite the greater magnitude of the relative antilipolytic effect of insulin, fat explants of rosiglitazone-treated rats continued to release more absolute amounts of NEFAs than control ING (Fig. 5c) or RETRO explants (Fig. 5g).

Quantification of major determinants of glycerol and NEFA re-esterification (GYK and PEPCK mRNA levels and activity of the respective proteins) and NEFA reuptake (FABP4 and FATP fatty acid transporter protein mRNA levels) indicated that lipolysis was stimulated by rosiglitazone, despite large increases in these processes, which
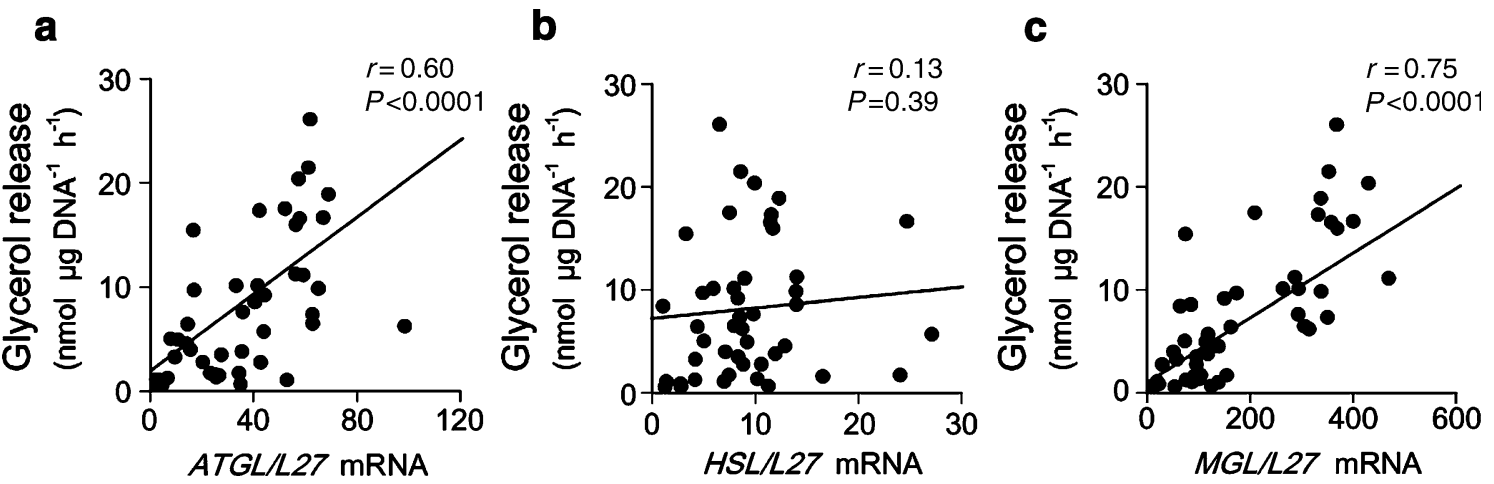

Fig. 3 Correlations between glycerol release and levels of $A T G L$ mRNA $(n=46, \mathbf{a}), H S L$ mRNA $(n=45$, b), and $M G L$ mRNA $(n=46$, c). The four adipose depots harvested from six control and six rosiglitazone-treated rats were included 


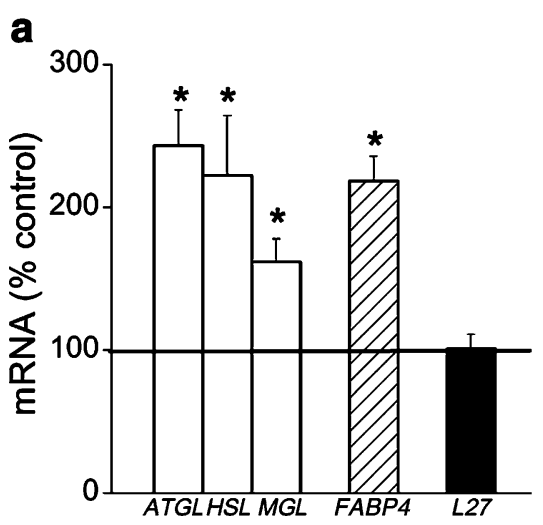

Fig. 4 Levels of $A T G L, H S L$ and $M G L$ mRNAs, expressed as percent of control, in ING (a) and RETRO (b) explants from untreated rats incubated in vitro with or without (horizontal line) rosiglitazone for 12

counteract the net release of lipolytic products, as further discussed in ESM text 5 and presented in ESM Fig. 2.

\section{Discussion}

This study investigated the impact and mechanisms of action of the thiazolidinedione rosiglitazone on WAT lipolysis and NEFA metabolism. Chronic rosiglitazone markedly induced WAT lipolysis, both under basal and stimulated conditions, and amplified the antilipolytic action of insulin under basal conditions. The study further revealed that rosiglitazone increases the levels of $A T G L$ and $M G L$ mRNAs in subcutaneous and visceral fat, and those of HSL mRNA in subcutaneous fat, suggesting that upregulation of lipase expression is part of the mechanisms of PPAR $\gamma$ action on adipose lipolysis.

The study confirmed the well-established actions of rosiglitazone on morphometric variables, indices of insulin sensitivity and lipidaemia [18-21]. Several lines of evidence suggest that thiazolidinediones exert their beneficial effects on whole-body insulin sensitivity partly by reducing circulating NEFAs and the consequent exposure of nonadipose tissues to the deleterious effects of NEFA metabolites on insulin signal transduction [2]. The present study demonstrates that, paradoxically to its effect on plasma NEFA, rosiglitazone markedly stimulates WAT lipolysis. Of note, the stimulatory action of rosiglitazone on lipolysis assessed in WAT explants and expressed per $\mu \mathrm{g}$ of DNA, thus correcting for cellularity, was maintained in isolated mature adipocytes. This excludes the possibility of the effect being due to the presence of a larger number of mature, lipolytically competent adipocytes having arisen through PPAR $\gamma$-induced adipocyte differentiation [22]. Thiazolidinediones exert depot-specific effects on fat accretion in humans [17], which is why several depots were investigated in this study. The findings show that the

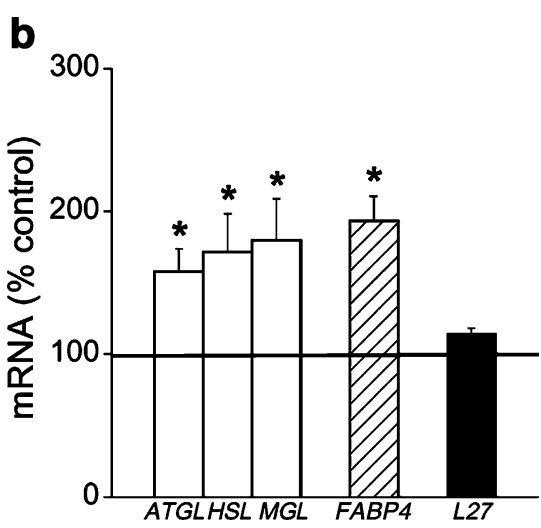

h. FABP4 and L27 mRNA served as positive and negative controls, respectively. Each column represents the mean $\pm \mathrm{SEM}$ of explants from four rats. ${ }^{*} p<0.05$ vs control explants

lipolysis-stimulating action of rosiglitazone extends to both subcutaneous and visceral adipose depots.

Studies that have addressed the effect of PPAR $\gamma$ agonism on adipose lipolysis have reported mixed results. Two studies found a reduction in the release of lipolytic products from rodent WAT explants and 3T3-L1 adipocytes $[5,6]$. These were, however, primarily designed to examine NEFA re-esterification under conditions that may have favoured the latter process. In contrast, studies that have specifically focused on in vivo NEFA kinetics in rats have shown that, in the fasting state, PPAR $\gamma$ agonists increase the capacity of WAT to release NEFAs [4, 7]. In addition, human adipocytes stimulated to differentiate in the presence of rosiglitazone displayed increased basal and noradrenaline-stimulated lipolysis [23]. In humans, the impact of PPAR $\gamma$ agonism on lipidaemia is more modest than in rodent models. Although some human in vivo turnover studies did not report any effect of PPAR $\gamma$ agonism on the kinetics of lipolytic products [24, 25], a net transcapillary release of NEFA from subcutaneous WAT was recently demonstrated in fasted type 2 diabetic subjects treated with rosiglitazone [26]. Taken together, these findings suggest that PPAR $\gamma$ agonism does stimulate the lipolytic process in the presence of low insulin concentrations.

Concomitant with lipolysis, rosiglitazone markedly increased mRNA levels of the lipases $A T G L$ and $M G L$ in all WAT depots, and those of HSL in the ING depot. A fairly robust correlation was found between basal rates of release of lipolytic products and mRNA levels of $A T G L$. This correlation obviously does not establish a cause and effect relationship; however, the importance of the expression level of $A T G L$ in WAT lipolysis is highlighted by recent studies in which $A T G L$ overexpression in 3T3-L1 adipocytes enhanced both fasting basal and isoproterenolstimulated lipolysis, whereas the opposite effects were found with inhibition of ATGL through antisense technology $[12,16,27]$. The functional importance of lipase 

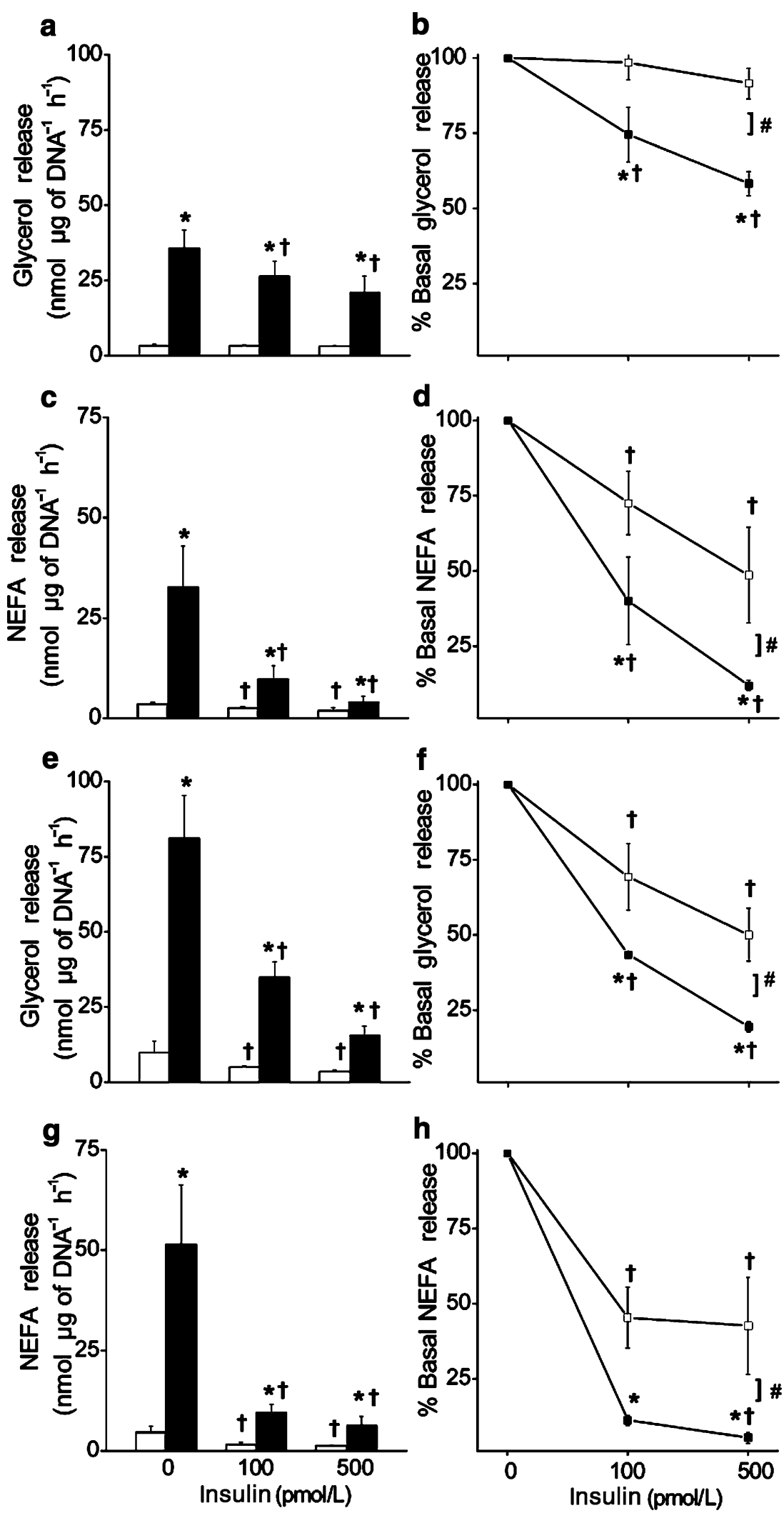

Fig. 5 Antilipolytic effect of insulin, expressed as absolute levels of glycerol and NEFA release $(\mathbf{a}, \mathbf{c}, \mathbf{e}, \mathbf{g})$ and per cent of basal (without insulin, $\mathbf{b}, \mathbf{d}, \mathbf{f}, \mathbf{h}$ ), on basal (unstimulated) glycerol and NEFA release, by explants of ING (a-d) and RETRO (e-h) adipose depots of control (open squares and columns) and rosiglitazone-treated rats (full squares

and columns). Each symbol or column represents the mean \pm SEM of six rats. ${ }^{*} p<0.05$ vs untreated control under the same incubation conditions; $\uparrow p<0.05$ vs without insulin in same treatment group; $\# p<0.05$ between control and rosiglitazone for dose-response curve analysed as a whole by ANOVA 
expression levels is further supported by the depot-specific behaviour of $A T G L$ mRNA levels and basal lipolysis in the present study: both were lower in ING than in visceral depots in control rats, but tended to respond more robustly to rosiglitazone, in relative terms, in the former than in the latter. This suggests that upregulation of $A T G L$ expression constitutes one mechanism by which PPAR $\gamma$ agonism enhances WAT basal lipolysis.

The mRNA levels of $M G L$ were also well correlated with the rosiglitazone-induced changes in lipolytic rates. Although recognised as an important enzyme for the complete hydrolysis of triglycerides in WAT [28], the modulation of $M G L$ has not received much attention. The finding that rosiglitazone increases $M G L$ mRNA levels in congruence with WAT basal lipolysis indicates that this enzyme may also be one of its regulated steps, as well as one additional level of action of PPAR $\gamma$ agonism thereupon.

With regard to $H S L$, other studies have reported a strong PPAR $\gamma$-induced increment in expression in human subcutaneous mature adipocytes [29] and in rodent brown adipocytes [30] incubated short-term with rosiglitazone. Also, HSL was recently shown to be a transcriptional target of PPAR $\gamma$ in differentiating pre-adipocytes and other tissues, but not in visceral WAT in vivo [31]. The present study revealed a short-term $(12 \mathrm{~h})$ induction of $H S L$ expression in vitro by rosiglitazone in both ING and RETRO depots, which persisted after a 7-day in vivo treatment in the ING but not in the RETRO depot. Taken together, these findings suggest depot and time dependence of the impact of PPAR $\gamma$ agonism on HSL expression. The fact that $H S L$ mRNA levels did not correlate with lipolysis, as did the other lipases, and that the absolute PPAR $\gamma$ mediated increase in lipolysis was quite similar in both basal and noradrenaline- or DBcAMP-stimulated conditions, leaves some uncertainty as to the contribution of $H S L$ to the PPAR $\gamma$ effect on lipolysis. Clearly, additional studies are needed to clarify this issue.

Under the present conditions, a direct action of rosiglitazone on expression of the lipases is supported by the observation that lipase mRNAs were increased within $12 \mathrm{~h}$ of in vitro exposure of naïve explants, as was the case for the PPAR response element-containing FABP4 gene. Such stimulation of the expression of intracellular lipases would efficiently complement the PPAR $\gamma$-mediated upregulation of perilipin expression [32, 33], which facilitates lipidlipase interactions and is a key player in the hormonal modulation of lipolysis. PPAR $\gamma$ agonism therefore appears to exert a multilevel, concerted action on the production of several key proteins, which results in an increase in the lipolytic capacity of adipose tissue.

To gain further insight into the consequences of PPAR $\gamma$ agonism on the physiological modulation of lipolysis, the effects of rosiglitazone on the response to lipolytic and antilipolytic hormones were investigated. Noradrenaline released by sympathetic nerve terminals in WAT is a major positive modulator of WAT lipolysis [34]. Glycerol and NEFA release reached under noradrenaline and DBcAMP stimulation were much higher in explants from rosiglitazone-treated than control rats. That DBcAMP was approximately as efficient as noradrenaline in stimulating lipolysis excludes the possibility of increased $\beta$-adrenergic receptor functionality and signal transduction as a mechanism of action of rosiglitazone on lipolysis. Such a conclusion is further supported by the mild downregulatory action of PPAR $\gamma$ agonism on $\beta_{3}$-receptor expression [35]. Instead, it appears that rosiglitazone increases levels of components of the lipolytic machinery (e.g. expression of lipases and other lipolysis-related proteins), thus allowing it to respond more robustly to adrenergic stimulation. Such amplification of the lipolytic potential by PPAR $\gamma$ agonism strikingly resembles that of the thermogenic potential reported in our previous study [35].

Insulin constitutes another major modulator of WAT lipolysis. The present study confirmed that rosiglitazone increases the response of WAT lipolysis to the inhibitory action of insulin [4]. It is worth noting that the inhibitory effect of insulin on NEFA was stronger than that on glycerol release, because of its additive effect on NEFA re-esterification to triglycerides [36, 37]. Despite the stronger antilipolytic effect of insulin on WAT lipolysis, explants of rosiglitazone-treated rats exposed to insulin concentrations approximating those found in vivo under fasting and postprandial conditions maintained a higher rate of NEFA release than controls. The apparent contradiction between this observation and the lower in vivo serum NEFA levels can be explained by the fact that, while reducing liver and muscle NEFA uptake, thiazolidinediones augment the ability of WAT to clear systemic NEFA through stimulation of protein-mediated NEFA uptake [3, 38]. The rosiglitazone-induced increase in FABP4 and FATP expression observed in the present study confirms previous studies $[39,40]$ and supports this notion. In vivo, the rosiglitazone-induced increase in WAT NEFA uptake and retention obviously overwhelmed that of net NEFA output, thus reducing plasma NEFAs. Additional potential in vivo contributors to NEFA lowering include the prevailing levels of antagonistic lipolytic modulators, regional blood flow, and NEFA uptake by brown adipose tissue (M. Laplante, Y. Deshaies, unpublished data). It should be noted that the robust stimulation of lipolysis by rosiglitazone occurred in the face of a large increase in the recycling of lipolytic products. Such futile cycling between lipolysis and re-esterification may have contributed to the enhanced response of lipolysis to hormonal modulation (see further discussion in ESM text 6). 
In conclusion, the results of this study establish that PPAR $\gamma$ activation increases basal lipolysis in WAT and amplifies its response to both adrenergic stimulation and insulin inhibition. The study further revealed that PPAR $\gamma$ agonism increases mRNA levels of $A T G L$ and $M G L$ in subcutaneous and visceral WAT, and that of $H S L$ in subcutaneous WAT, apparently via a direct action on gene expression. Given the importance of lipase expression levels on lipolytic activity, the findings suggest that, at least in the case of $A T G L$ and $M G L$, increased expression contributes to the positive action of PPAR $\gamma$ agonism on adipose lipolysis. In the face of increased lipolysis, the net release of NEFAs into the bloodstream is counteracted by increased reuptake and cycling. PPAR $\gamma$ agonism therefore reduces net NEFA release into the circulation while greatly increasing lipid substrate turnover, an action likely contributing to improve the hormonal fine tuning of adipose lipid metabolism.

Acknowledgements This work was supported by a grant from the Canadian Institutes of Health Research (CIHR) to Y. Deshaies. W. T. Festuccia was the recipient of a postdoctoral fellowship from a CIHR Training in Obesity Program grant. M. Laplante was the recipient of a studentship from the Natural Sciences and Engineering Research Council of Canada. The authors are very grateful for the invaluable professional assistance of J. Lalonde, M. Alain and S. Poulin. They also thank K. Cianflone and P. Mauriège for their helpful review of the manuscript.

Duality of interest The authors declare that there is no duality of interest associated with this study.

\section{References}

1. Fürnsinn C, Waldhäusl W (2002) Thiazolidinediones: metabolic actions in vitro. Diabetologia 45:1211-1223

2. Kintscher U, Law RE (2005) PPAR $\gamma$-mediated insulin sensitization: the importance of fat versus muscle. Am J Physiol Endocrinol Metab 288:E287-E291

3. Ye JM, Dzamko N, Cleasby ME et al (2004) Direct demonstration of lipid sequestration as a mechanism by which rosiglitazone prevents fatty-acid-induced insulin resistance in the rat: comparison with metformin. Diabetologia 47:1306-1313

4. Oakes ND, Thalen PG, Jacinto SM, Ljung B (2001) Thiazolidinediones increase plasma-adipose tissue FFA exchange capacity and enhance insulin-mediated control of systemic FFA availability. Diabetes 50:1158-1165

5. Guan HP, Li Y, Jensen MV, Newgard CB, Steppan CM, Lazar MA (2002) A futile metabolic cycle activated in adipocytes by antidiabetic agents. Nat Med 8:1122-1128

6. Tordjman J, Chauvet G, Quette J, Beale EG, Forest C, Antoine B (2003) Thiazolidinediones block fatty acid release by inducing glyceroneogenesis in fat cells. J Biol Chem 278:18785-18790

7. Kalderon B, Mayorek N, Ben-Yaacov L, Bar-Tana J (2003) Adipose tissue sensitization to insulin induced by troglitazone and MEDICA 16 in obese Zucker rats in vivo. Am J Physiol Endocrinol Metab 284:E795-E803

8. Fredrikson G, Belfrage P (1983) Positional specificity of hormone-sensitive lipase from rat adipose tissue. J Biol Chem 258:14253-14256
9. Fortier M, Wang SP, Mauriege P et al (2004) Hormone-sensitive lipase-independent adipocyte lipolysis during beta-adrenergic stimulation, fasting, and dietary fat loading. Am J Physiol Endocrinol Metab 287:E282-E288

10. Haemmerle G, Zimmermann R, Hayn M et al (2002) Hormonesensitive lipase deficiency in mice causes diglyceride accumulation in adipose tissue, muscle, and testis. J Biol Chem 277: 4806-4815

11. Okazaki H, Osuga J, Tamura Y et al (2002) Lipolysis in the absence of hormone-sensitive lipase: evidence for a common mechanism regulating distinct lipases. Diabetes 51:3368-3375

12. Zimmermann R, Strauss JG, Haemmerle G et al (2004) Fat mobilization in adipose tissue is promoted by adipose triglyceride lipase. Science 306:1383-1386

13. Villena JA, Roy S, Sarkadi-Nagy E, Kim KH, Sul HS (2004) Desnutrin, an adipocyte gene encoding a novel patatin domaincontaining protein, is induced by fasting and glucocorticoids: ectopic expression of desnutrin increases triglyceride hydrolysis. J Biol Chem 279:47066-47075

14. Jenkins CM, Mancuso DJ, Yan W, Sims HF, Gibson B, Gross RW (2004) Identification, cloning, expression, and purification of three novel human calcium-independent phospholipase $A_{2}$ family members possessing triacylglycerol lipase and acylglycerol transacylase activities. J Biol Chem 279:48968-48975

15. Zechner R, Strauss JG, Haemmerle G, Lass A, Zimmermann R (2005) Lipolysis: pathway under construction. Curr Opin Lipidol $16: 333-340$

16. Kershaw EE, Hamm JK, Verhagen LA, Peroni O, Katic M, Flier JS (2006) Adipose triglyceride lipase: function, regulation by insulin, and comparison with adiponutrin. Diabetes 55:148-157

17. Mori Y, Murakawa Y, Okada K et al (1999) Effect of troglitazone on body fat distribution in type 2 diabetic patients. Diabetes Care 22:908-912

18. Picard F, Auwerx J (2002) PPAR $\gamma$ and glucose homeostasis. Annu Rev Nutr 22:167-197

19. Willson TM, Lambert MH, Kliewer SA (2001) Peroxisome proliferator-activated receptor gamma and metabolic disease. Annu Rev Biochem 70:341-367

20. Laplante M, Sell H, MacNaul KL, Richard D, Berger JP, Deshaies Y (2003) PPAR-gamma activation mediates adipose depotspecific effects on gene expression and lipoprotein lipase activity: mechanisms for modulation of postprandial lipemia and differential adipose accretion. Diabetes 52:291-299

21. Berthiaume M, Sell H, Lalonde J et al (2004) Actions of PPARgamma agonism on adipose tissue remodeling, insulin sensitivity, and lipemia in absence of glucocorticoids. Am J Physiol Regul Integr Comp Physiol 287:R1116-R1123

22. Tontonoz P, Hu E, Spiegelman BM (1994) Stimulation of adipogenesis in fibroblasts by PPAR gamma 2, a lipid-activated transcription factor. Cell 79:1147-1156

23. van Harmelen V, Dicker A, Ryden M et al (2002) Increased lipolysis and decreased leptin production by human omental as compared with subcutaneous preadipocytes. Diabetes 51:2029-2036

24. Mayerson AB, Hundal RS, Dufour S et al (2002) The effects of rosiglitazone on insulin sensitivity, lipolysis, and hepatic and skeletal muscle triglyceride content in patients with type 2 diabetes. Diabetes 51:797-802

25. Racette SB, Davis AO, McGill JB, Klein S (2002) Thiazolidinediones enhance insulin-mediated suppression of fatty acid flux in type 2 diabetes mellitus. Metabolism 51:169-174

26. Tan GD, Fielding BA, Currie JM et al (2005) The effects of rosiglitazone on fatty acid and triglyceride metabolism in type 2 diabetes. Diabetologia 48:83-95

27. Lake AC, Sun Y, Li JL et al (2005) Expression, regulation, and triglyceride hydrolase activity of adiponutrin family members. J Lipid Res 46:2477-2487 
28. Fredrikson G, Tornqvist H, Belfrage P (1986) Hormone-sensitive lipase and monoacylglycerol lipase are both required for complete degradation of adipocyte triacylglycerol. Biochim Biophys Acta 876:288-293

29. McTernan PG, Harte AL, Anderson LA et al (2002) Insulin and rosiglitazone regulation of lipolysis and lipogenesis in human adipose tissue in vitro. Diabetes 51:1493-1498

30. Teruel T, Hernandez R, Rial E, Martin-Hidalgo A, Lorenzo M (2005) Rosiglitazone up-regulates lipoprotein lipase, hormonesensitive lipase and uncoupling protein-1, and down-regulates insulin-induced fatty acid synthase gene expression in brown adipocytes of Wistar rats. Diabetologia 48:1180-1188

31. Deng T, Shan S, Li P-P et al (2005) Peroxisome proliferatoractivated receptor gamma transcriptionally up-regulates hormonesensitive lipase via the involvement of Sp1. Endocrinology 147:875-884

32. Dalen KT, Schoonjans K, Ulven SM et al (2004) Adipose tissue expression of the lipid droplet-associating proteins S3-12 and perilipin is controlled by peroxisome proliferator-activated receptor-gamma. Diabetes 53:1243-1252

33. Rosenbaum SE, Greenberg AS (1998) The short- and long-term effects of tumor necrosis factor- $\alpha$ and BRL 49653 on peroxisome proliferator-activated receptor (PPAR) $\gamma 2$ gene expression and other adipocyte genes. Mol Endocrinol 12:1150-1160
34. Bartness TJ, Kay Song C, Shi H, Bowers RR, Foster MT (2005) Brain-adipose tissue cross talk. Proc Nutr Soc 64:53-64

35. Sell H, Berger JP, Samson P et al (2004) Peroxisome proliferatoractivated receptor $\gamma$ agonism increases the capacity for sympathetically mediated thermogenesis in lean and $o b / o b$ mice. Endocrinology 145:3925-3934

36. Rosenstock M, Greenberg AS, Rudich A (2001) Distinct longterm regulation of glycerol and non-esterified fatty acid release by insulin and TNF-alpha in 3T3-L1 adipocytes. Diabetologia 44:55-62

37. Reshef L, Olswang Y, Cassuto H et al (2003) Glyceroneogenesis and the triglyceride/fatty acid cycle. J Biol Chem 278:30413-30416

38. Coort SL, Coumans WA, Bonen A, van der Vusse GJ, Glatz JF, Luiken JJ (2005) Divergent effects of rosiglitazone on proteinmediated fatty acid uptake in adipose and in muscle tissues of Zucker rats. J Lipid Res 46:1295-1302

39. Frohnert BI, Hui TY, Bernlohr DA (1999) Identification of a functional peroxisome proliferator-responsive element in the murine fatty acid transport protein gene. J Biol Chem 274:39703977

40. Tontonoz P, Hu E, Graves RA, Budavari AI, Spiegelman BM (1994) mPPAR gamma 2: tissue-specific regulator of an adipocyte enhancer. Genes Dev 8:1224-1234 